\title{
Paving the way to daytime optical feeder links based on LGS assisted adaptive optics
}

D. Alaluf, M. Centrone, D. Bonaccini Calia, P. Suetterling, R. Speziali, et al.

D. Alaluf, M. Centrone, D. Bonaccini Calia, P. Suetterling, R. Speziali, M. Faccini, W. Hackenberg, J. Perdigues Armengol, A. Di Paola, "Paving the way to daytime optical feeder links based on LGS assisted adaptive optics," Proc. SPIE 11852, International Conference on Space Optics - ICSO 2020, 118525Y (11 June 2021); doi: 10.1117/12.2600028

SPIE Event: International Conference on Space Optics - ICSO 2021, 2021, Online Only 


\section{International Conference on Space Optics-ICSO 2020}

Virtual Conference

30 March-2 April 2021

Edited by Bruno Cugny, Zoran Sodnik, and Nikos Karafolas
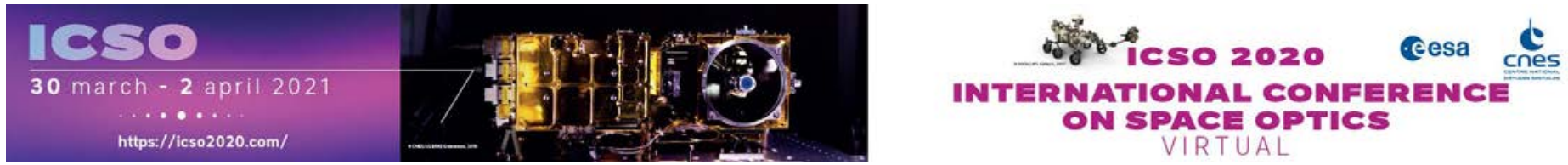

\section{Paving the way to daytime optical feeder links based on LGS assisted adaptive optics}

\section{Cesa isoporeseatings denes}




\title{
Paving the way to Daytime Optical Feeder Links based on LGS assisted Adaptive Optics
}

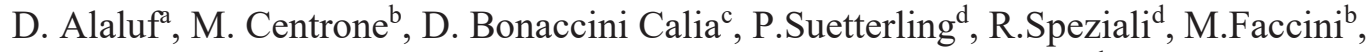 \\ W.Hackenberg ${ }^{\mathrm{c}}$, J.Perdigues Armengol ${ }^{\mathrm{a}}$, A.Di Paola ${ }^{\mathrm{b}}$
}

\author{
${ }^{a}$ European Space Agency (ESA)/ESTEC, 2200AG Noordwijk, The Netherlands; \\ ${ }^{b}$ INAF Osservatorio Astronomico di Roma, Via Frascati 33, 00078 Monte Porzio Catone (Roma), \\ Italy; ' European Southern Observatory, Karl-Schwarzschildstr. 2, 85748 Garching, Germany; \\ ${ }^{\mathrm{d} I n s t i t u t e}$ for Solar Physics, AlbaNova University Center, SE-106 91 Stockholm, Sweden
}

\begin{abstract}
We report on experiments performed with the Swedish Solar Telescope (SST) located in La Palma (Canary Islands) to observe the sodium Laser Guide Star (LGS) during daytime. The ESO Wendelstein Laser Guide Star Unit 20W CW $589 \mathrm{~nm}$ laser was used to generate an LGS plume at Earth's Mesosphere, the laser launch telescope being at a distance of $92 \mathrm{~m}$ from the SST, where a special daytime receiver was installed. The photometry of the LGS plume and the background effects were measured as a function of the angular separation between the plume and the Sun. The ultimate goal is to demonstrate closed-loop LGS Adaptive Optics (AO) during daytime, for Optical Feeder Satellite Communications operating 24/7, as well as for astrophysics of the Sun Coronal Mass Ejections. Other applications of daytime LGS-AO are for thermal imaging of astrophysical objects. This paper presents several system aspects of the daytime LGS-AO setup and discusses the experimental results obtained. We conclude that using an ultra-narrow band magneto-optical filter installed in the daytime SST receiver, the sky background flux is very efficiently suppressed and the LGS flux is well sufficient to drive LGS-AO during daytime for correction at visible wavelengths.
\end{abstract}

Keywords: Free space optical communications, Optical feeder links, Laser Guide Star, Daytime observing, Adaptive Optics.

\section{INTRODUCTION}

Optical feeder links (OFL) have become more and more popular in response to a growing demand for high-speed communications terminals. Optical communications is of particular interest, with respect to radio frequency communications, in terms of available bandwidth, low mass, low volume, power consumption and data transfer security. Furthermore, no frequency license is required to operate optical communications since laser beams do not lead to interferences between different data links [1].

Despite its great potential, some challenging aspects need to be addressed to exploit this technology. Indeed, laser-based communications suffer multiple distortions when crossing the atmosphere. These distortions not only impact the Bit Error Ratio (BER) of the system but can even make the link unfeasible [2]. Most of the modern astronomical telescopes are equipped with Adaptive Optics (AO) systems, making it possible to estimate and compensate the atmospheric turbulences disturbing the observations. To do so, a bright beacon located nearby the object of interest is often required, to be used as a reference for the wavefront compensation. In some cases, Natural Guide Stars (NGS) can be used, provided that they are close enough from the scientific target to undergo a similar atmospheric distortion. Obviously, this constraint imposes a severe limitation on the targets that can be observed. In the case of ground-tosatellite optical uplinks, one might consider to use a beacon transmitted by the satellite (i.e the downlink signal), as a reference to pre-compensate the (uplink) beam transmitted to the satellite. In this way, the pre-compensation counteracts the atmospheric distortion experienced by the uplink beam when crossing the atmosphere. This approach, however, might not work properly due to the satellite motion which implies that the uplink transmission beam has to go through a 
slightly different atmosphere patch than the downlink satellite reference beam, so called "point-ahead angle" (PAA). Hence, using the satellite signal as a reference might be partially efficient in the GEO case (which involves a PAA of typically 4arcsec), but most likely not for ground-to-LEO optical links (which involves a PAA of about 10arcsec) [3].

Future optical feeder link satellites networks [4,5] will require a more reliable approach to cope with atmospheric distortions occurring during OFL. An alternative consists in using the Laser Guide Stars (LGS) approach, a key technology of AO used in astronomy, to create an artificial beacon in the desired line of sight [6-8]. Sodium LGS take advantage of the natural Sodium atomic layer in the upper atmosphere (between $85 \mathrm{~km}$ and $100 \mathrm{~km}$ altitude). A powerful laser is tuned to excite the D2 line of the Sodium atoms of the mesosphere (at about 589nm in air); the photons are absorbed by the Sodium atoms, then spontaneously emitted, hence creating an artificial beacon. This beacon can then be used as reference for the uplink pre-compensation, without any limitation related to the PAA.

Optical feeder links during daytime are even more challenging than during nighttime since $i$ ) the atmospheric turbulences are stronger than during the night and ii) the (weak) useful signal is completely overwhelmed by the much stronger sky background noise. In astrophysics the usefulness of daytime AO is for science cases at thermal wavelengths [9], imaging and spectroscopy, for observations to be executed in the first hours, and for solar physics observations at the solar limb chromosphere and corona, for example during coronal mass ejection events. The present paper discusses the use of LGS beacon for daytime operation. We report on an experiment conducted in October 2019 using the Swedish Solar Telescope (SST) located in La Palma (Canary Islands), to observe the Sodium LGS during daytime. A special receiver, using an ultra narrow-band Magneto-Optical filter to reject the sky background, was installed at the focal plane of the SST, located $92 \mathrm{~m}$ away from the laser launch telescope. The photometry of the LGS plume and the background effects were measured as a function of the angular separation between the LGS plume and the Sun.

In the framework of the ongoing ESO-ESA R\&D cooperation, the demonstration of LGS-AO at visible wavelength using Pyramid WFS and optical feeder uplink pre-compensation is being prepared, to be installed at the ESA 1m Optical Ground Station (Tenerife). This is the CaNaPy project, done in collaboration with several institutes and industries. Refer to this conference presentation and proceeding for more details. Thanks to the assessed feasibility of daytime LGS detection presented in this paper, CaNaPy will include a demonstration of daytime LGS-AO operation for SatComm. The reported experiment aims at establishing the Signal to Noise Ratio (SNR) of the LGS which will be seen in monostatic propagation of the laser beam from OGS.

The section 2 of the paper describes the setup used for the experiment and section 3 introduces the methodology used for the data reduction and presents the experimental results obtained.

\section{EXPERIMENTAL SETUP}

The ESO Wendelstein Laser Guide Star Unit (WLGSU) [10], located in front of the William Herschel Telescope, was used to propagate during daytime a $300 \mathrm{~mm}$ laser beam at a power of $19.3 \mathrm{~W} \mathrm{CW}$, with a circular polarization emission and a D2b repumping line at 10\% relative intensity. The laser launch telescope was protected with an Aluminium layer to protect it from daytime heat. The Swedish Solar Telescope, located about 92m away from the WLGSU, was used to star at the LGS light from the side (bistatic configuration), hence creating an elongated 'plume' LGS of 54.5" on the detector. The experimental configuration is shown in Figure 1. A dedicated receiver was designed, assembled and installed in the SST laboratory to observe the LGS plume during daytime. Its main optical components consist of a quarter waveplate to linearize the polarization of the LGS photons, a $0.5 \mathrm{~nm}$ FWHM pre-filter, a $100 \mathrm{~m} \AA$ FWHM ultranarrow band Magneto-Optical Filter (MOF) with its crossed-polarizers (intrinsic throughput of 97\% \& 100 000:1 extinction ratio), and image scaling optics creating a focal plane for the ZWO ASI1600pro camera, with an image scale of $0.06 \% /$ pixel and a format of 1600x1600 pixels. The pre-filter and the ultra-narrow band MOF, both centered at 589nm, were used to filter out the sky background from the plume measurements (Figure 2).

The experiment started once the Sun was out of the laser launch telescope unvignetted angle, to prevent damaging the optics of the WLGSU. The laser was pointed at a fixed position in the Sky (at ALT 85.8, AZ -60.9), in the field of view of the SST, which was also keeping a fixed pointing direction. The plume SNR and the sky background were measured at different time intervals (hence also for various angular separation between the plume and the Sun since the LGS plume and the SST were fixed, while the Sun was moving). 
a)

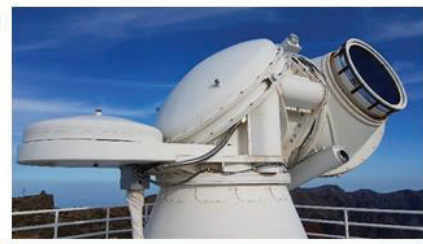

b)

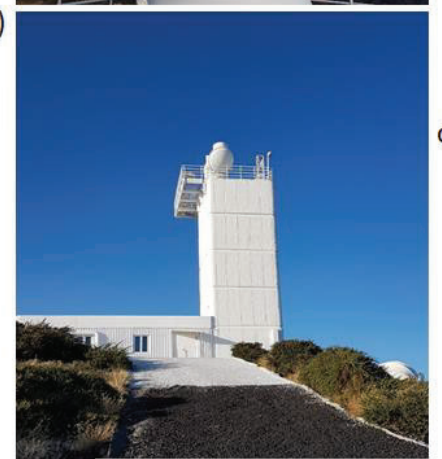

Swedish Solar Telescope

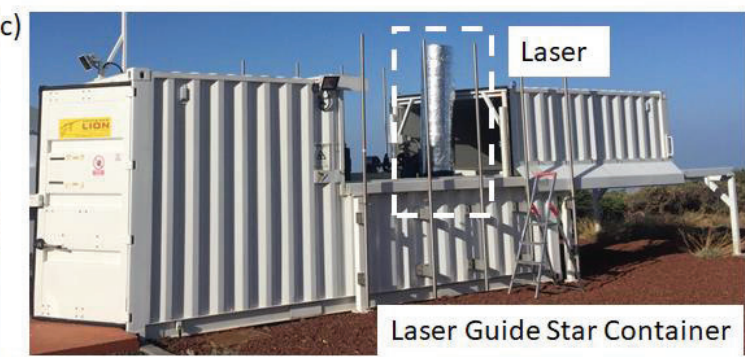

d)

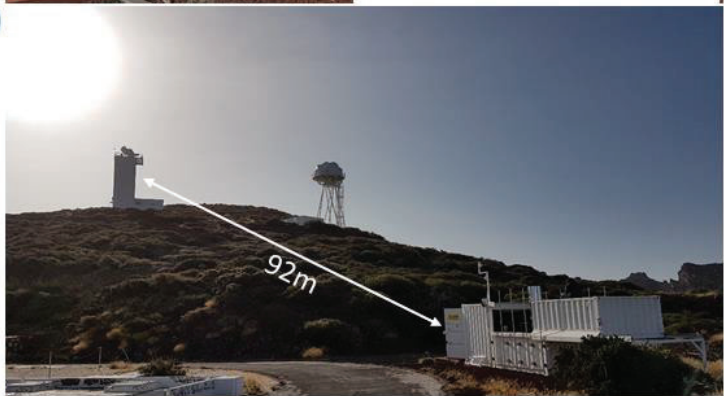

Figure 1 Experimental configuration. a) and b) show the Swedish Solar Telescope (SST) where the daytime receiver was installed, c) shows the Wendelstein Laser Guide Star Unit (WLGSU) used to create a reference beacon for atmospheric turbulence compensation, d) shows both the SST and the WLGSU, separated by a distance of $92 \mathrm{~m}$.

LEGEND

a)

1. $10 \mathrm{~mm}$ Diaphragm at SST focus (field stop)

2. $589 \mathrm{~nm}$ Quarterwaveolate on mount

3. $589 \mathrm{~nm}$ laser mirror $\mathrm{AOI} 45$, polarization preserving

4. $589 \mathrm{~nm}$ laser mirror $\mathrm{AOI}, 45$ polarization preserving

5. $200 \mathrm{~mm}$ EFL on $30 \mathrm{~mm}$ cage mount

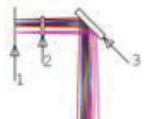

6. Narrow band $(0.6 \mathrm{~nm})$ Na Filter mounted on MOF

7. $-500 \mathrm{~mm}$ EFL lens at Pupil (SST L1) on mount

8. $100 \mathrm{~mm}$ EFL on mount, with micrometric Focus adjuster

9. Image plane, SBIG camera ( or iXon Camera)

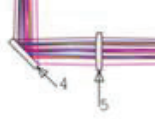

MOF with MOF with
Polarizers

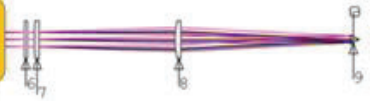

b)
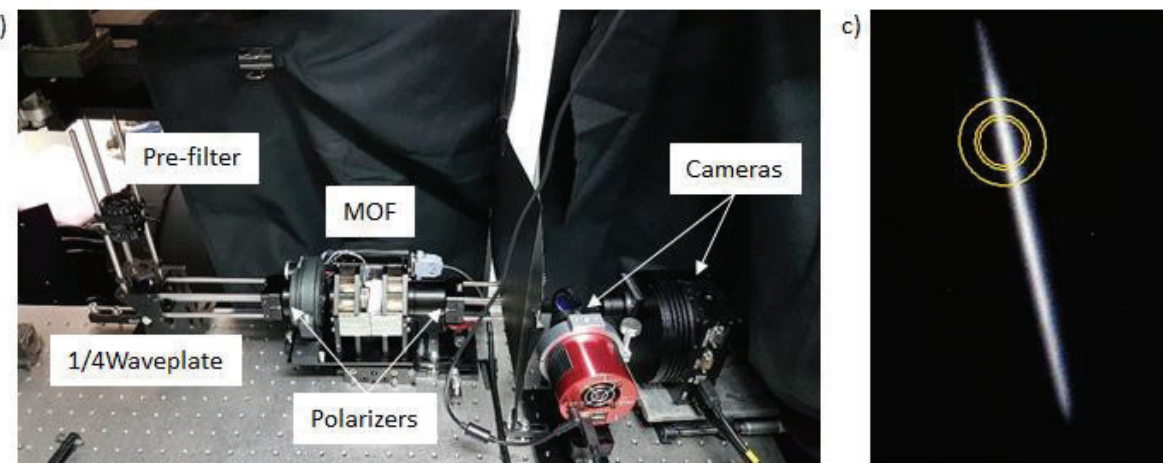

Figure 2 a) Optical design of the daytime receiver installed at the focal plane of the Swedish Solar Telescope, b) Experimental setup, c) Example of elongated plume as seen with the SST due to the bistatic configuration of the experiment (i.e the laser was propagated and observed using two separated telescopes). 


\section{EXPERIMENTAL RESULTS}

The measurements were performed on the $30^{\text {th }}$ October 2019 from 10.00 am to $2.00 \mathrm{pm}$, using various integration times (namely $3 \mathrm{~s}, 1 \mathrm{~s}, 100 \mathrm{~ms}$ and $20 \mathrm{~ms}$ ). For each integration time, sequences of 50 frames of the plume were recorded together with the corresponding calibration frames used for the data reduction (flat fields, bias frames, dark frames). Figure 3 shows an example of a measurement performed with an integration time of $20 \mathrm{~ms}$. Panel a) shows the raw image acquired with the camera; one note the plume can barely be distinguished from the background. The background is not from the sky, but mostly the bias from the camera. Applying a cascade of two median filters makes it possible to reveal it with respect to the background (panel b) ). Then, a segmentation algorithm was used to identify the plume area and a cutoff was applied to remove non-physical branches as shown on panel c). A systematic frame filtering method was used to reject the plumes featuring unusual relative dimensions. Finally, an elliptic mask was fitted to the shape of the plume as shown on panel d). This approach was used for each frame of the sequence and for each frame set related to an integration time. The photometry of the sky background and that of the plume as a function of the time (or equivalently as a function of the angular separation between the LGS and the Sun) were then extracted.

a)

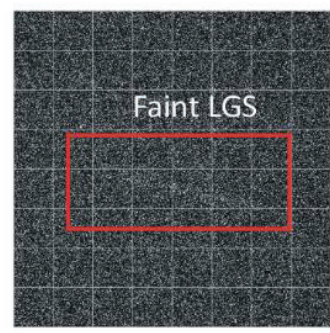

b)

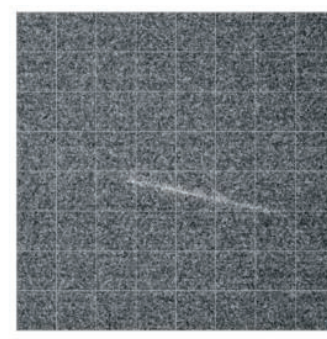

c)

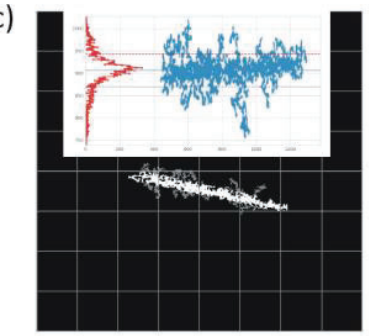

d)

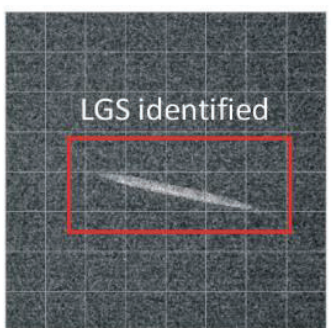

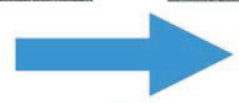

Median

Filtering

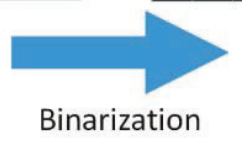

Rotation \& cutoff

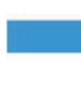

Derotation

Elliptic mask

Figure 3. Acquisition with an integration time of $20 \mathrm{~ms}$. The LGS is initially very faint but appears thanks to image processing: two median filters are applied in cascade to remove the noise, followed by a binarization to identify the plume with respect to the sky background. Then, a cutoff is applied to eliminate non-physical branches on the plume. Finally, an elliptic mask is applied to fit the plume shape. Note that we could not use a specialized, ultra low readout noise camera for the experiment. At these frame rates the readout noise was around $100 \mathrm{ADU}$.

Thin cirrus clouds have affected the measurements, giving increased background at different times in the measurement days. The variability of the sky background, even with thin cirrus clouds, still allows the needed LGS SNR to be achieved. Even in this worst-case scenario results are good. We have been able to detect the plume with good SNR at $20 \mathrm{~ms}$ integration time. Note that the ZWO camera readout noise at fast frame rates was consistently around 100 ADU.

As opposed to the (bistatic) configuration used for the present experiment, producing an elongated plume of 54.5"x2.4" FWHM on the detector, the CaNaPy system to be installed at ESA's OGS will be used in monostatic configuration (i.e the laser will be propagated and observed with the same telescope pupil), hence the LGS will look like a round spot star on the detector. We present hereafter the main results obtained integrating numerically the LGS plume into a round shape LGS. The sky background is computed from the same images as the LGS plume. The LGS integrated flux was measured to be $23.5 \times 10^{6}$ photons $/ \mathrm{s} / \mathrm{m}^{2}$, which corresponds e.g. to 235 photons $/ \mathrm{ms} /$ subaperture for a Shack Hartmann wavefront sensor featuring 10x10 subapertures running at $1 \mathrm{kHz}$ with a $1 \mathrm{~m}$ Telescope, and to 18 photons/ms/pixel on a Pyramid WFS with 40x40 pixels pupils. The LGS return flux obtained after sky subtraction thanks to the magnetooptical filter are comparable to those obtained during nighttime experiments.

The results demonstrating the feasibility of using the LGS in daytime are shown in Figure 4. A SNR was defined as the ratio of the flux in the integrated LGS plume vs the Sky background, measured on the same image, both integrated over 
an area corresponding to the circular LGS, 2.4" FWHM, as it will be seen from the ESA OGS in monostatic Propagation under worse seeing conditions.
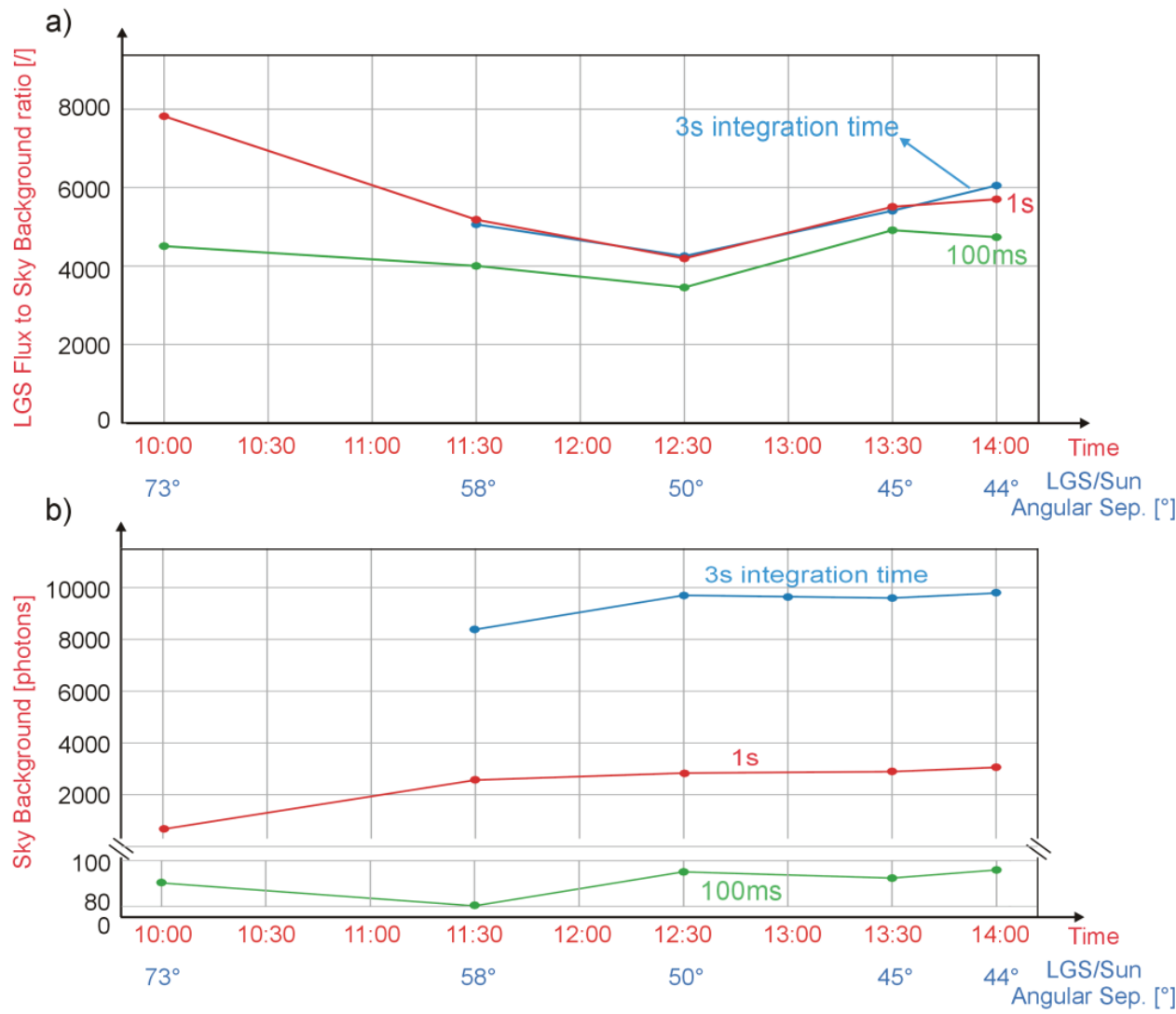

Figure 4 Figure a) shows the ratio between the integrated flux over the LGS plume area and the sky background, which can be considered a SNR useful to assess the feasibility of LGS sensing in daytime. Figure b) shows the sky background photometry, measured on the same frame as the LGS image. Both quantities are presented as a function of the time of the day as well as of the angular separation between the Sun and the LGS. Measurements taken for integration times of $3 \mathrm{~s}$, $1 \mathrm{~s}$ and $100 \mathrm{~ms}$. Cirrus clouds have been present at times, and are responsible for trend variations.

\section{CONCLUSION}

A successful experiment was conducted to characterize the feasibility of the LGS-AO during daytime. The ultimate goal is to demonstrate closed-loop LGS-AO during daytime, for optical feeder satellite communications operating 24/7, as well as for astrophysics applications (e.g sun corona observations, coronal mass ejections, daytime thermal bands observations of astrophysical objects). The results show that for the ESA OGS, we should expect an LGS flux SNR, vs the sky background, of about 400 with $10 \mathrm{~ms}$ integration time. The results also show that in daytime the LGS flux at the ground is similar to nighttime. We conclude that using the MOF filter, the sky background flux is very efficiently suppressed, even in thin cirrus conditions; the LGS flux is well sufficient to drive LGS-AO in daytime for adaptive optics corrections at visible wavelengths. In an operational scenario, we have learned how the launch telescope and the instrumentation should be shielded from sunlight. 


\section{ACKNOWLEDGEMENT}

The experiment was carried out in the framework of the ongoing ESA-ESO R\&D cooperation with the support from INAF (Osservatorio Astronomico di Roma) and from the Swedish Solar Telescope of the Institute for Solar Physics of Stockholm University. The authors gratefully acknowledge Prof. G.B.Scharmer from the SST and the Instituto de Astrofisica de Canarias (IAC) for their support during the measurements campaign. D. Alaluf was supported by a PostDoc grant provided by the ScyLight programme.

\section{REFERENCES}

[1] H . Henniger, O. Wilfert, An Introduction to Free-space Optical Communications. Radioengineering, 19(2).

[2] A. Belmonte, Taylor, M. T., Hollberg, L., \& Kahn, J. M. (2017). Effect of atmospheric anisoplanatism on earthto-satellite time transfer over laser communication links. Optics express, 25(14), 15676-15686, 2010.

[3] Alaluf, D., JM Perdigues Armengol, and Z. Sodnik. "How effective is tip-tilt pre-compensation for optical uplinks based on the received downlink optical signal?." International Conference on Space Optics-ICSO 2018. Vol. 11180. International Society for Optics and Photonics, 2019.

[4] Hauschildt, Harald, et al. "ESAs ScyLight programme: Activities and status of the high throughput Optical Network" HydRON"." International Conference on Space Optics-ICSO 2018. Vol. 11180. 2019.

[5] Hauschildt, Harald, et al. "HydRON: High throughput optical network." 2019 IEEE International Conference on Space Optical Systems and Applications (ICSOS). IEEE, 2019.

[6] Foy, R., and A. Labeyrie. "Feasibility of adaptive telescope with laser probe." Astronomy and Astrophysics 152 (1985): L29-L31.

[7] Fugate, Robert Q., et al. "Measurement of atmospheric wavefront distortion using scattered light from a laser guide-star." Nature 353.6340 (1991): 144-146.

[8] Primmerman, C. A., and D. V. Murphy. "Page DA et al. 1991." Nature 353: 141.

[9] J.M.Beckers, " Daytime Observations with ELTs in the Thermal Infrared Using Laser Guide Star Adaptive Optics", in Proc. of the AO4ELT Conference, 2011 (http://ao4elt2.lesia.obspm.fr/spip.php\%3Farticle734.html)

[10] Calia, D. Bonaccini, et al. "The ESO transportable LGS Unit for measurements of the LGS photon return and other experiments." Modern Technologies in Space-and Ground-based Telescopes and Instrumentation II. Vol. 8450. International Society for Optics and Photonics, 2012. 